\title{
BESSEL SERIES EXPANSIONS OF THE EPSTEIN ZETA FUNCTION AND THE FUNCTIONAL EQUATION
}

\author{
AUDREY A. TERRAS
}

ABSTRACT. For the Epstein zeta function of an $n$-ary positive definite quadratic form, $n-1$ generalizations of the Selberg-Chowla formula (for the binary case) are obtained. Further, it is shown that these $n-1$ formulas suffice to prove the functional equation of the Epstein zeta function by mathematical induction. Finally some generalizations of Kronecker's first limit formula are obtained.

1. Introduction. Let $S$ be the $n \times n$ matrix of a positive definite (real) quadratic form and let $\rho$ be a complex variable with $\operatorname{Re} \rho>1 / 2 n$. Then Epstein's zeta function is defined by

$$
Z_{n}(S, \rho)=\frac{1}{2} \sum_{a \in Z^{n}-0}\left({ }^{t} a S a\right)^{-\rho},
$$

where the sum is over all column vectors with integral coordinates, not all of which are zero. In the following, for any matrix $A$, the transpose of $A$ will be denoted ${ }^{t} A$. Epstein [6] proved the analytic continuation, functional equation, and Kronecker limit formula for (1.1). The latter is the computation of the constant term in the Laurent expansion of the function about $\rho=1 / 2 n$. Applications of the Kronecker limit formula to algebraic number theory are given by Hecke [7, p. $198 \mathrm{ff}$ ] and Siegel [13, pp. 93 and 117].

We shall reprove Epstein's result and, in fact, show that there are $n-1$ different ways of obtaining limit formulas. Our point of departure is a formula to be found in papers of Selberg-Chowla [11, formulas (5) and (6), p. 87] and Bateman-Grosswald [3, Theorem 1, p. 366]:

$$
\begin{gathered}
Z_{2}(S, \rho)=s_{2}^{-\rho} \zeta(2 \rho)+t^{1 / 2-\rho} s_{2}^{-1 / 2} \pi^{1 / 2} \Gamma(\rho-1 / 2) \Gamma(\rho)^{-1} \zeta(2 \rho-1) \\
+2 \pi^{1 / 2} \Gamma(\rho)^{-1} t^{1 / 2-\rho} s_{2}^{-1 / 2} \sum_{a \geq 1 ; b \neq 0}\left(\pi t^{1 / 2} s_{2}^{-1 / 2} a|b|\right)^{\rho-1 / 2} a^{1-2 \rho} \cos (2 \pi q b a) \\
\times K_{1 / 2-\rho}\left(2 \pi t^{1 / 2} s_{2}^{-1 / 2} a|b|\right),
\end{gathered}
$$

Presented to the Society, November 18, 1972; received by the editors May 5, 1972. AMS (MOS) subject classifications (1970). Primary $10 \mathrm{H} 10$.

Key words and phrases. Epstein zeta function, Chowla-Selberg formula, functional equation, Bessel functions, Dedekind $\eta$-function, Kronecker limit formula. 
where

$$
\begin{gathered}
S=\left(\begin{array}{ll}
s_{1} & s_{12} \\
s_{12} & s_{2}
\end{array}\right)=\left(\begin{array}{ll}
1 & q \\
0 & 1
\end{array}\right)\left(\begin{array}{ll}
t & 0 \\
0 & s_{2}
\end{array}\right)\left(\begin{array}{ll}
1 & 0 \\
q & 1
\end{array}\right) \\
\text { for } t=s_{1}-q^{2} s_{2}=s_{2}^{-1}\left(s_{1} s_{2}-s_{12}^{2}\right)>0 \text { and } q=s_{12} / s_{2} \text {. }
\end{gathered}
$$

This is the diagonalization of Siegel [12, p. 24]. Also $\zeta(x)$ is Riemann's zeta function, and $K_{\nu}(z)$ is the modified Bessel function of the second kind defined by

$$
K_{\nu}(z)=\frac{1}{2} \int_{0}^{\infty} \exp \{-z(u+1 / u) / 2\} u^{\nu-1} d u, \text { for }|\arg z|<1 / 2 \pi .
$$

Formula (1.2) gives the analytic continuation of $Z_{2}$ and the functional equation as is proved in Bateman-Grosswald [3, p. 366, remarks after statement of Theorem 1]. Moreover (1.2) gives a good approximation of $Z_{2}$ in the critical strip [3, Theorems 2,3 , pp. 366-367]. And it can be shown by the same method that we use in $\$ 3$ of this paper that (1.2) implies the Kronecker limit formula (Siegel [12, pp. 5-17]):

$$
\begin{aligned}
& \lim _{\rho \rightarrow 1}\left\{Z_{2}(S, \rho)-1 / 2 \pi|S|^{-1 / 2}(\rho-1)^{-1}\right\} \\
& \quad=\pi|S|^{-1 / 2}\left\{\gamma-\log 2-\log \left(t^{1 / 2}|\eta(z)|^{2}\right)\right\} .
\end{aligned}
$$

Here $|S|=$ determinant of $S, \gamma=$ Euler's constant, and $\eta(z)$ is the Dedekind $\eta$ function defined by

$$
\eta(z)=\exp \{i \pi z / 12\} \prod_{b \geq 1}(1-\exp \{2 \pi i z b\}), \quad \operatorname{Im} z>0 .
$$

Also, $t$ and $q$ being defined as in (1.3), we set

$$
z=q+i t^{1 / 2} s_{2}^{-1 / 2}=s_{2}^{-1}\left(s_{12}+i|S|^{1 / 2}\right),
$$

which is a well-defined element of the upper half plane, since $S$ is a positive definite symmetric matrix. Thus $z$ is the usual element of the upper half plane associated to the positive quadratic form $S$ (Siegel $[13$, p. 6] or Leveque $[9$, p. 16]).

In $\$ 2$, we shall generalize (1.2) to $Z_{n}$ in $n-1$ different ways. The only results needed for this are the Poisson summation formula for $\left(\mathbf{R}^{n}, \mathbf{Z}^{n}\right)$ and an integral formula relating the Fourier transform of a radial function in $\mathbf{R}^{n}$ to the Hankel transform (Stein-Weiss [14, Theorem 3.3, p. 155]). One of the $n-1$ formulas was obtained by Berndt [4, formula (5.2)] by other methods. And Epstein himself has such a formula [6, formula (10), p. 641]. However the single formulas of Epstein and Berndt do not seem to suffice to prove the functional equation. We shall prove that any correctly chosen pair of the $n-1$ formulas does imply the functional equation. 
In $\$ 3$, generalizations of the Kronecker limit formula (1.5) are considered. Of the $n-1$ formulas which we have generalizing (1.2) it seems that only the formula of Epstein himself produces an $(n-1)$-fold product generalizing $\eta(z)$ because of the simple form of $K_{1 / 2}(z)=(\pi / 2 z)^{1 / 2} e^{-z}$. Since generalizations of the Kronecker limit formula should contain "automorphic forms" generalizing the Dedekind $\eta$ function, one is lead to consider the more general definition of automorphic forms given by Borel [5, p. 200]. One sees that the Epstein zeta function and its generalizations [15] are themselves automorphic forms in this sense. In fact they are what Borel calls "Eisenstein series" [5, Example 2, p. 209]. Thus one would expect the coefficients in the Laurent expansion of $Z_{n}(S, \rho)$ about $\rho=1 / 2 n$ to have similar behavior to $Z_{n}(S, \rho)$. That is, the coefficients, considered as functions on the space $P_{n}$ of positive definite symmetric matrices, are invariant with respect to the transformations of $P_{n}$ given by $S \mapsto S[U]$, for $S$ in $P_{n}$ and $U$ an integral matrix of determinant \pm 1 . Further, when invariant differential operators on $P_{n}[15$, pp. 176-177] are applied to the coefficients individually a finite dimensional vector space should be generated. This last property of the coefficients is condition (2) in Borel's definition of automorphic form essentially [5, p. 200]. However this is not the place to discuss the matter in detail.

Also the question of extending the results even to the zeta functions of one complex variable considered in [15] remains open. The simplest cases seem to be complicated and must require results on Bessel functions of matrix argument of Herz [8].

2. Expressions for $Z_{n}$ in a series of Bessel functions. First some notation must be set up. Let $n=n_{1}+n_{2}$, with $1 \leq n_{i} \leq n-1$. And decompose $S$ in block matrix form, as Siegel does [12, pp. 24-25]:

$$
S^{(n)}=\left(\begin{array}{ll}
S_{1}^{\left(n_{1}\right)} & S_{12} \\
t_{12} & S_{2}^{\left(n_{2}\right)}
\end{array}\right)=\left(\begin{array}{ll}
I & { }^{t} Q \\
0 & I
\end{array}\right)\left(\begin{array}{ll}
T^{\left(n_{1}\right)} & 0 \\
0 & S_{2}^{\left(n_{2}\right)}
\end{array}\right)\left(\begin{array}{ll}
I & 0 \\
Q & I
\end{array}\right)
$$

where $I$ denotes the identity matrix and 0 denotes the zero matrix. We shall sometimes write $T_{S}$ (or $T$ ) in place of $T^{\left(n_{1}\right)}$ and $Q_{S}$ in place of $Q$. Here we use the notation $S^{(n)}$ for an $n \times n$ matrix and drop the $(n)$ if no confusion is likely. We shall also write $S[A]={ }^{t} A S A$, and $|S|=$ determinant of $S$. Define

$$
H_{n_{1}, n_{2}}(S, \rho)=\left|S_{2}\right|^{-1 / 2} \sum_{a \in Z^{n_{1}}-0 ; b \in Z^{n_{2}}-0}(T[a])^{1 / 4 n_{2}-1 / 2 \rho}\left(S_{2}^{-1}[b]\right)^{1 / 2 \rho-1 / 4 n_{2}}
$$

$$
\times \exp \left\{2 \pi i^{t} b Q a\right\} K_{1 / 2 n}-\rho\left(2 \pi\left(T[a] S_{2}^{-1}[b]\right)^{1 / 2}\right)
$$


where the sum runs over integral column vectors $a$ and $b$ not all of whose entries are zero.

Theorem 1. $H_{n_{1}, n_{2}}$ is an entire function of the complex variable $\rho$, and, for $\operatorname{Re} \rho>1 / 2 n$,

$$
\begin{aligned}
& \pi^{-\rho} \Gamma(\rho) Z_{n}(S, \rho) \\
& =\pi^{-\rho} \Gamma(\rho) Z_{n_{2}}\left(S_{2}, \rho\right)+\pi^{1 / 2 n_{2}}-\rho\left(\rho-1 / 2 n_{2}\right)\left|S_{2}\right|^{-1 / 2} Z_{n_{1}}\left(T, \rho-1 / 2 n_{2}\right) \\
& +H_{n_{1}, n_{2}}(S, \rho) \text {. }
\end{aligned}
$$

Here $Z_{1}(S, \rho)=s^{-\rho} \zeta(2 \rho)$, where $\zeta$ is Riemann's zeta function.

Proof. A column vector in $\mathrm{Z}^{n}$ may be considered as composed of 2 parts $\left(\begin{array}{l}a \\ b\end{array}\right)$ with $a$ in $\mathbf{Z}^{n_{1}}$ and $b$ in $\mathbf{Z}^{n_{2}}$. Then, using (2.1), $S[(\underset{b}{a})]=T[a]+S_{2}[Q a+b]$. It follows that

$$
\begin{aligned}
Z_{n}(S, \rho) & =\frac{1}{2} \sum_{\left(\begin{array}{c}
a \\
b
\end{array}\right) \neq\left(\begin{array}{l}
0 \\
0
\end{array}\right)} S\left[\left(\left(\begin{array}{c}
a \\
b
\end{array}\right)\right]^{-\rho}=\frac{1}{2} \sum_{a=0 ; b \neq 0}+\frac{1}{2} \sum_{a \neq 0 ; \text { all } b \text { in } Z^{n_{2}}}\right. \\
& =Z_{n_{2}}\left(S_{2}, \rho\right)+\frac{1}{2} \sum_{a \in Z^{n_{1}}-0 ; \text { all } b \in Z^{n_{2}}}\left(T[a]+S_{2}[Q a+b]\right)^{-\rho} .
\end{aligned}
$$

Now apply Poisson summation with respect to the sum over $b$. Then the second term in the expression of $Z_{n}$ is

$$
\frac{1}{2} \sum_{a \in Z^{n} 1-0 ; b \in Z^{n_{2}}} \int_{\mathbf{R}^{n_{2}}}\left(T[a]+S_{2}[Q a+x]\right)^{-\rho} \exp \left\{-2 \pi i^{t} b x\right\} d x,
$$

where $x$ is a column vector in $\mathbf{R}^{n_{2}}$ and the integral is the Lebesgue integral in $\mathbf{R}^{n_{2}}$. Now make the change of variables $y=(T[a])^{-1 / 2} W(Q a+x)$, where $S_{2}={ }^{t} W W$. Then the integral over $\mathbf{R}^{n_{2}}$ is seen to be

$$
(T[a])^{1 / 2 n} 2^{-\rho}\left|S_{2}\right|^{-1 / 2} \exp \left\{2 \pi i^{t} b Q a\right\} I_{n_{2}}\left(2 \pi(T[a])^{1 / 2 t}\left({ }^{t} b W^{-1}\right), \rho\right),
$$

where we define

$$
I_{n}(b, \rho)=\int_{\mathbf{R}^{n}}\left(1+{ }^{t} x x\right)^{-\rho} \exp \left\{-i^{t} b x\right\} d x, \quad \operatorname{Re} \rho>1 / 2 n .
$$

Now $I(0, \rho)$ is evaluated easily by induction. For set

$$
t^{2}=1+x_{1}^{2}+\cdots+x_{n-1}^{2} \text { and } x_{n}=t y .
$$

Then changing variables to $\left(x_{1}, \cdots, x_{n-1}, y\right)$, we see that

$$
I_{n}(0, \rho)=\int\left(1+y^{2}\right)^{-\rho} t^{1-2 \rho} d x_{1} \cdots d x_{n-1} d y=I_{1}(0, \rho) I_{n-1}(0, \rho-1 / 2) \text {. }
$$


And it is easy to see that $I_{1}(0, \rho)=\Gamma(\rho-1 / 2) \Gamma(1 / 2) / \Gamma(\rho)$, so that induction finishes the proof of the formula:

$$
I_{n}(0, \rho)=\pi^{1 / 2 n} \Gamma(\rho-1 / 2 n) / \Gamma(\rho) .
$$

If $b \neq 0, I_{n}(b, \rho)$ is evaluated using the fact proved in Stein and Weiss [14, Theorem 3.3 , p. 155] that the Fourier transform of a rotation invariant or radial function in $\mathbf{R}^{n}$ can be reduced to a Hankel or Bessel transform in one-dimensional space. The result is

$$
I_{n}(b, \rho)=\int_{0}^{\infty}\left(1+u^{2}\right)^{-\rho} u^{n-1}(2 \pi)^{1 / 2 n} J_{1 / 2 n-1}(|b| u)(|b| u)^{1-1 / 2 n} d u,
$$

for $b \neq 0$. Here $J$ denotes the Bessel function of the first kind:

$$
J_{\nu}(x)=(1 / 2 x)^{\nu} \pi^{-1 / 2} \Gamma(\nu+1 / 2)^{-1} \int_{0}^{\pi} \exp \{-i x \cos t\} \sin ^{2 \nu} t d t, \quad \operatorname{Re} \nu>-1 / 2 .
$$

Then using a formula from Abramowitz-Stegun [1, formula. (11.4.44), p. 488] one obtains

$$
I_{n}(b, \rho)=2 \pi^{1 / 2 n} \Gamma(\rho)^{-1}(1 / 2\|b\|)^{\rho-1 / 2 n} K_{1 / 2 n-\rho}(\|b\|),
$$

for $b \neq 0$ where $\|b\|=\left({ }^{t} b \cdot b\right)^{1 / 2}$. Here $K$ is the modified Bessel function of the second kind defined in (1.4).

The proof is now complete except to note that $H_{n_{1}, n_{2}}$ is entire in this case for the same reason as in the paper of Bateman-Grosswald [3, Lemma 2]. So the analytic continuation of $Z_{n}$ is a consequence of formula (2.3).

The remainder of this section is devoted to a proof that the $n-1$ formulas for $Z_{n}$ given in (2.3) imply the functional equation of $Z_{n}$. The method is simply induction on $n$ and some matrix identities.

Theorem 2. Define $R_{n}(S, \rho)=\pi^{-\rho} \Gamma(\rho) Z_{n}(S, \rho)$. Then the Epstein zeta.function bas the following functional equation:

$$
R_{n}(S, \rho)=|S|^{-1 / 2} R_{n}\left(S^{-1}, 1 / 2 n-\rho\right) .
$$

Proof (Induction on $n$ ). The functional equation for the case $n=1$ is that of Riemann's zeta function, which is well known. We now proceed by mathematical induction and assume the functional equation for all $Z_{k}$, where $1 \leq k \leq n-1$. Then (2.3) may be written:

$$
\begin{aligned}
\pi^{-\rho} \Gamma(\rho) Z_{n}(S, \rho)= & \pi^{-\rho} \Gamma(\rho) Z_{r}\left(S_{2}, \rho\right) \\
& +\left|S_{2}\right|^{-1 / 2} \pi^{-(\rho-1 / 2 r)} \Gamma(\rho-1 / 2 r) Z_{n-r}(T, \rho-1 / 2 r)+H_{n-r, r}(S, \rho) .
\end{aligned}
$$

We may use the functional equation of $Z_{r}$ to rewrite the middle term of the righthand side to obtain 
$\pi^{-\rho} \Gamma(\rho) Z_{n}(S, \rho)=\pi^{-\rho} \Gamma(\rho) Z_{r}\left(S_{2}, \rho\right)$.

$$
+|S|^{-1 / 2} \pi^{-(1 / 2 n-\rho)} \Gamma(1 / 2 n-\rho) Z_{n-r}\left(T^{-1}, 1 / 2 n-\rho\right)+H_{n-r, r}(S, \rho) .
$$

Here we have used the fact that $|S|=\left|S_{2}\right| \cdot|T|$, an easy consequence (2.1) when $n_{1}=r$ and $n_{2}=n-r$.

Now we know that $Z_{n}(S[U], \rho)=Z_{n}(S, \rho)$ for any unimodular matrix $U$, that is, any matrix $U$ with integral entries and determinant +1 or -1 . And we shall show at the end of this proof that for the particular unimodular matrix

$$
U=\left(\begin{array}{ll}
0 & I^{(n-r)} \\
I^{(r)} & 0
\end{array}\right)
$$

the following matrix identities hold:

$$
\begin{gathered}
\left(S^{-1}[U]\right)_{2}=\left(T_{S}\right)^{-1}, \\
\left(T_{S^{-1}[U]}\right)^{-1}=S_{2}, \\
{ }^{t} Q_{S^{-1}[U]}=-Q_{S} .
\end{gathered}
$$

Therefore, replacing $S$ by $S^{-1}[U]$ and $\rho$ by $1 / 2 n-\rho$ in (2.6), we see that

$$
\begin{aligned}
&|S|^{-1 / 2} \pi^{-(1 / 2 n-\rho)} \Gamma(1 / 2 n-\rho) Z_{n}\left(S^{-1}, 1 / 2 n-\rho\right) \\
&=|S|^{-1 / 2} \pi^{-(1 / 2 n-\rho)} \Gamma(1 / 2 n-\rho) Z_{n}\left(S^{-1}[U], 1 / 2 n-\rho\right) \\
&=|S|^{-1 / 2} \pi^{-(1 / 2 n-\rho)} \Gamma(1 / 2 n-\rho) Z_{r}\left(\left(S^{-1}[U]\right)_{2}, 1 / 2 n-\rho\right) \\
&+\pi^{-\rho} \Gamma(\rho) Z_{n-r}\left(\left(T-1, S^{-1}[U)^{-1}, \rho\right)+|S|^{-1 / 2} H_{n-r, r}\left(S^{-1}[U], 1 / 2 n-\rho\right)\right. \\
&=|S|^{-1 / 2} \pi^{-(1 / 2 n-\rho)} \Gamma(1 / 2 n-\rho) Z_{r}\left(\left(T S^{-1}, 1 / 2 n-\rho\right)\right. \\
&+\pi^{-\rho} \Gamma(\rho) Z_{n-r}\left(S_{2}, \rho\right)+H_{r, n-r}(S, \rho) .
\end{aligned}
$$

Here we have used formulas (2.7)-(2.9) as well as the definition (2.2) of $H_{n-r, r}$, plus the fact that for the modified Bessel function of the second kind $K_{\nu}=K_{-\nu}$.

Therefore by (2.6) with $r$ replaced by $n-r$, we see that

$$
|S|^{-1 / 2} \pi^{-(1 / 2 n-\rho)} \Gamma(1 / 2 n-\rho) Z_{n}\left(S^{-1}, 1 / 2 n-\rho\right)=\pi^{-\rho} \Gamma(\rho) Z_{n}(S, \rho) .
$$

This is of course the functional equation, and we now see that only two of the formulas (2.3) are required to prove it. However, one does not seem to suffice.

It only remains to prove the identities (2.7)-(2.9). We should note a certain abuse of notation occurring here. For $S_{2}$ and $T_{S}$ both depend upon $r$ as well as $S$. However the formulas would be awkward if we did not omit this dependence.

The proofs of formulas $(2.7-2.8)$ are straightforward matrix computations using (2.1) and the following property of the matrix $U$ : 


$$
\text { if } S=\left(\begin{array}{ll}
S_{1}^{(n-r)} & S_{12} \\
{ }^{t} S_{12} & S_{2}^{(r)}
\end{array}\right), \quad \text { then } S[U]=\left(\begin{array}{ll}
S_{2} & { }^{t} S_{12} \\
S_{12} & S_{1}
\end{array}\right) \text {. }
$$

Now (2.1) implies

$$
S^{-1}=\left(\begin{array}{ll}
\left(T_{S}\right)^{-1} & 0 \\
0 & \left(S_{2}\right)^{-1}
\end{array}\right)\left[\left(\begin{array}{ll}
1 & -Q_{S} \\
0 & I
\end{array}\right)\right]=\left(\begin{array}{cc}
\left(T_{S}\right)^{-1} & -\left(T_{S}\right)^{-1}{ }^{t} Q_{S} \\
* & *
\end{array}\right) .
$$

So

$$
S^{-1}[U]=\left(\begin{array}{cc}
* & * \\
* & \left(T_{S}\right)^{-1}
\end{array}\right)
$$

Then (2.7) is clear. And (2.8) follows from (2.7) upon replacing $S$ by $\left(S^{-1}\right)\left[{ }^{t} U\right]$. Note that

$$
{ }^{t} U=\left(\begin{array}{ll}
0 & I^{(r)} \\
I^{(n-r)} & 0
\end{array}\right)=U^{-1} .
$$

For (2.9) compute $S^{-1}[U]$ in two ways. The above gives

$$
S^{-1}[U]=\left(\begin{array}{cc}
* & * \\
-\left(T_{S}\right)^{-1}{ }^{t} Q_{S} & *
\end{array}\right)
$$

Multiply out (2.1) to obtain

$$
S^{-1}[U]=\left(\begin{array}{cc}
* & * \\
\left(S^{-1}[U]\right)_{2} Q_{S^{-1}[U]} & *
\end{array}\right)
$$

This completes the proof.

3. Generalizations of Kronecker's limit formula. The $n-1$ formulas (2.3) yield $n-1$ generalizations of the Kronecker limit formula, of which one is the formula obtained by Epstein [6, p. 644].

Definition. $k_{n}(S)=\lim _{\rho \rightarrow 1 / 2 n}\left\{Z_{n}(S, \rho)-1 / 2 \pi^{1 / 2 n} \Gamma(1 / 2 n)^{-1}|S|^{-1 / 2}(\rho-1 / 2 n)^{-1}\right\}$.

Note that this is just the constant term in the Laurent expansion of the Epstein zeta function about the pole $\rho=1 / 2 n$. This is a consequence of the fact that the residue of the Epstein zeta function at $\rho=1 / 2 n$ is

$$
1 / 2|S|^{-1 / 2} \pi^{1 / 2 n} \Gamma(1 / 2 n)^{-1} \text {. }
$$

Epstein [6, pp. 623-627] has shown the above formula for the residue by a method generalizing Riemann's. The formula may also be proved from (2.3) and the fact that the residue. of the Riemann zeta function $\zeta(s)$ at $s=1$ is 1 . This generalizes 
an argument of Bateman-Grosswald for the case of binary quadratic forms $[3$, remarks after Theorem 1, p. 366].

\section{Theorem 4.}

$$
\begin{aligned}
k_{n}(S)= & Z_{n_{2}}\left(S_{2}, 1 / 2 n\right)+\Gamma\left(1 / 2 n_{1}\right) \Gamma(1 / 2 n)^{-1} k_{n_{1}}\left(T^{\left(n_{1}\right)}\right)\left|S_{2}\right|^{-1 / 2} \pi^{1 / 2 n_{2}} \\
& +H_{n_{1}, n_{2}}(S, 1 / 2 n) \pi^{1 / 2 n} \Gamma(1 / 2 n)^{-1}+1 / 2|S|^{-1 / 2} \pi^{1 / 2 n} \Gamma(1 / 2 n)^{-1}\left\{\psi\left(1 / 2 n_{1}\right)-\psi(1 / 2 n)\right\},
\end{aligned}
$$

where $\psi(z)=\Gamma^{\prime}(z) \Gamma(z)^{-1}$ and

$$
\begin{aligned}
H_{n_{1}, n_{2}}(S, 1 / 2 n) & \\
=\left|S_{2}\right|^{-1 / 2} \sum_{a \in Z^{n_{1}}-0 ; b \in Z^{n_{2}-0}} & \exp \left\{2 \pi i^{t} b Q a\right\}\left(S_{2}^{-1}[b]\right)^{1 / 4 n} 1(T[a])^{-1 / 4 n} 1 \\
& \times K_{1 / 2 n_{1}}\left(2 \pi\left(T[a] S_{2}^{-1}[b]\right)^{1 / 2}\right) .
\end{aligned}
$$

Proof. This is an easy computation using the formula (2.3). One need only note that if we set

$$
\Gamma\left(\rho-1 / 2 n_{2}\right) \Gamma(\rho)^{-1}=a_{0}+a_{1}(\rho-1 / 2 n)+\cdots,
$$

then

$$
a_{0}=\Gamma\left(1 / 2 n_{1}\right) \Gamma(1 / 2 n)^{-1} \quad \text { and } \quad a_{1}=\Gamma\left(1 / 2 n_{1}\right) \Gamma(1 / 2 n)^{-1}\left\{\psi\left(1 / 2 n_{1}\right)-\psi(1 / 2 n)\right\}
$$

And let the Laurent expansion of $Z_{n_{1}}\left(T, \rho-1 / 2 n_{2}\right)$ about $\rho=1 / 2 n$ be

$$
Z_{n_{1}}\left(T, \rho-1 / 2 n_{2}\right)=b_{-1}(\rho-1 / 2 n)^{-1}+b_{0}+\cdots \cdot
$$

Then by the definition and remarks above,

$$
b_{0}=k_{n_{1}}(T) \text { and } \quad b_{-1}=1 / 2|T|^{-1 / 2} \pi^{1 / 2 n_{1}} \Gamma\left(1 / 2 n_{1}\right)^{-1} .
$$

Clearly the constant term in the Laurent expansion of the middle term of the righthand side of (2.3) about $\rho=1 / 2 n$ is $\left|S_{2}\right|^{-1 / 2} \pi^{1 / 2 n} 2\left(a_{0} b_{0}+a_{1} b_{-1}\right)$. Thus the constant term in the Laurent expansion of the entire right-hand side of (2.3) about $\rho=1 / 2 n$ is

$$
k(S)=Z_{n_{2}}\left(S_{2}, 1 / 2 n\right)+\left|S_{2}\right|^{-1 / 2} \pi^{1 / 2 n}\left(a_{0} b_{0}+a_{1} b_{-1}\right)+H_{n_{1}, n_{2}}(S, 1 / 2 n) \pi^{1 / 2 n} \Gamma(1 / 2 n)^{-1}
$$

Substituting the values of $a_{0}, a_{1}, b_{-1}, b_{0}$ into the above equation, we obtain Theorem 4. We have included the formula for $H_{n_{1}, n_{2}}(s, 1 / 2 n)$ in the statement of the theorem for reference. It is an immediate result of the definition (2.2) and the that $K_{\nu}=K_{-\nu}$.

Corollary (Epstein [6, p. 644]). Let $n_{1}=1$ and $T^{(1)}=T_{S}=t$ in (2.1). And define 


$$
\begin{aligned}
z\{b\} & ={ }^{t} b Q+i t^{1 / 2}\left(S_{2}^{-1}[b]\right)^{1 / 2}, \\
c_{n} & = \begin{cases}\gamma-\log 2-1 / 2 \sum_{r=1}^{1 / 2 n-1} r^{-1}, & \text { for } n \text { even, } \\
y-\sum_{r=0}^{1 / 2(n-3)}(2 r+1)^{-1}, & \text { for } n \text { odd } .\end{cases}
\end{aligned}
$$

Then

$$
\begin{aligned}
k(S)= & Z_{n-1}\left(S_{2}, 1 / 2 n\right) \\
+ & \pi^{1 / 2 n}\left|S_{2}\right|^{-1 / 2} \Gamma(1 / 2 n)^{-1} \\
& \times\left\{c_{n}-\log \left(\left.t^{1 / 2}\left\|_{b \in Z^{n-1}-0 ; b(\bmod \pm 1)}(1-\exp \{2 \pi i z\{b\}\})\right\|\right|^{2}\right)\right\}
\end{aligned}
$$

$B y " b(\bmod \pm 1)$ ", we mean that $b$ runs over a complete set of representatives for the equivalence relation $b \sim b^{\prime}$ when $b=( \pm 1) b$ '. And " \|\| " in the infinite product denotes the usual absolute value of a complex number.

Proof. Take $n_{1}=1$ in the preceding theorem.

First recall that in the one-dimensional case $Z_{1}(t, \rho)=t^{-\rho} \zeta(2 \rho)$, where $\zeta$ is Riemann's zeta function. The formula $k_{1}(t)=t^{-1 / 2}\left(\gamma-\log t^{1 / 2}\right)$ is a consequence of the following expansions about $\rho=1 / 2$ :

$$
t^{-\rho}=t^{-1 / 2}-(\log t) t^{-1 / 2}(\rho-1 / 2)+\cdots, \quad \zeta(2 \rho)=(2 \rho-1)^{-1}+\gamma+\cdots,
$$

where $\gamma$ is Euler's constant.

Second, use Abramowitz-Stegun [1, formulas (6.3.2) and (6.3.4), p. 258] to see that

$$
\psi(1 / 2)-\psi(1 / 2 n)= \begin{cases}-2 \log 2-\sum_{r=1}^{1 / 2 n-1} r^{-1}, & \text { for } n \text { even, } \\ -2 \sum_{r=1}^{1 / 2(n-3)}(2 r+1)^{-1}, & \text { for } n \text { odd. }\end{cases}
$$

Third, since $K_{1 / 2}(z)=(\pi / 2 z)^{1 / 2} e^{-z}$, it follows that

$$
\begin{gathered}
H_{1, n-1}(S, 1 / 2 n)=1 / 2|S|^{-1 / 2} \sum_{a \in Z-0 ; b \in Z^{n-1}-0}\|a\|^{-1} \exp \left\{2 \pi i\left({ }^{t} b Q a+i t^{1 / 2}\left(S_{2}^{-1}[b]\right)^{1 / 2}\|a\|\right)\right\} . \\
=|S|^{-1 / 2} \sum_{a \geq 1 ; b \in Z^{n-1}-0 ; b(\bmod \pm 1)}\left(a^{-1} \exp \{2 \pi i z\{b\} a\}+\overline{\left.a^{-1} \exp \{2 \pi i z\{b\} a\}\right)} .\right.
\end{gathered}
$$


Here "__ denotes the complex conjugate. Now apply the expansion $\log (1-w)=-\sum_{n=1}^{\infty} n^{-1} w^{n}$ for $\|w\|<1$, to obtain the desired result:

$$
H_{1, n-1}(S, 1 / 2 n)=|S|^{-1 / 2} \log \left\|\prod_{b \in Z^{n-1}-0 ; b(\bmod \pm 1)}(1-\exp \{2 \pi i z\{b\}\})\right\|^{2} \cdot
$$

The proof of the corollary is complete when we substitute the expressions for $k(t), \psi(1 / 2)-\psi(1 / 2 n), H_{1, n-1}(S, 1 / 2 n)$ into the formula of Theorem 4 .

Note that the corollary gives a function which generalizes the Dedekind $\eta$ function (1.6) in a more obvious way than the result of Theorem 4. However the analogous function in Theorem 4 must also generalize the $\eta$-function in some way which may be fruitful recalling the results of Asai [2] and Koecher [10] as well as the general definition of automorphic forms given by Borel [5, p. 200]. But much work remains to be done in this direction.

\section{REFERENCES}

1. M. Abramowitz and I. A. Stegun (Editors), Handbook of mathematical functions, with formulas, graphs and mathematical tables, 3rd printing, with corrections, Nat. Bur. Standards Appl. Math. Series, 55, Superintendent of Documents, U. S. Government Printing Office, Washington, D. C., 1965. MR 31 \#1400.

2. T. Asai, On a certain function analogous to $|\log \eta(z)|$, Nagoya Math. J. 40 (1970), 193-211. MR 42 \#5921.

3. P. T. Bateman and E. Grosswald, On Epstein's zeta function, Acta Arith. 9 (1964), 365-373. MR 31 \#3392.

4. B. C. Berndt, Identities involving the coefficients of a class of Dirichlet series. VI, Trans. Amer. Math. Soc. 160 (1971), 157-167. MR 43 \#6412.

5. A. Borel and G. Mostow (Editors), Algebraic groups and discontinuous subgroups, Proc. Sympos. Pure Math., vol. 9, Amer. Math. Soc., Providence, R. I., 1966.

6. Paul Epstein, Zur Theorie allgemeiner Zetafunktionen. I, Math. Ann. 56 (1903), 614-644.

7. E. Hecke, Mathematische Werke, Vandenhoeck \& Ruprecht, Göttingen, 1959. MR 21 \#3303.

8. C. S. Herz, Bessel functions of matrix argument, Ann. of Math. (2) 61 (1955), 474523. MR 16, 1107.

9. W. J. Leveque, Topics in number theory. Vol II, Addison-Wesley, Reading, Mass., 1956. MR 18, 283.

10. M. Koecher, Ein neuer Beweis der Kroneckerschen Grenzformel, Arch. Math. 4 (1953), 316-321. MR 15, 507.

11. A. Selberg and S. Chowla, On Epstein's zeta function, J. Reine Angew. Math. 227 (1967), 86-110. MR 35 \#6632.

12. C. L. Siegel, Lectures on quadratic forms, Tata Inst., Bombay, 1957,

13. - Lectures on advanced analytic number theory, Tata Inst., Bombay, 1961.

14. E. M. Stein and G. Weiss, Introduction to Fourier analysis on Euclidean spaces, Princeton Univ. Press, Princeton, N. J., 1971.

15. A. Terras, A generalization of Epstein's zeta function, Nagoya Math. J. 42 (1971), 173-188. MR $44 \# 3970$.

DEPARTMENT OF MATHEMATICS, UNIVERSITY OF CALIFORNIA AT SAN DIEGO, LA JOLLA, CALIFORNIA 92037 